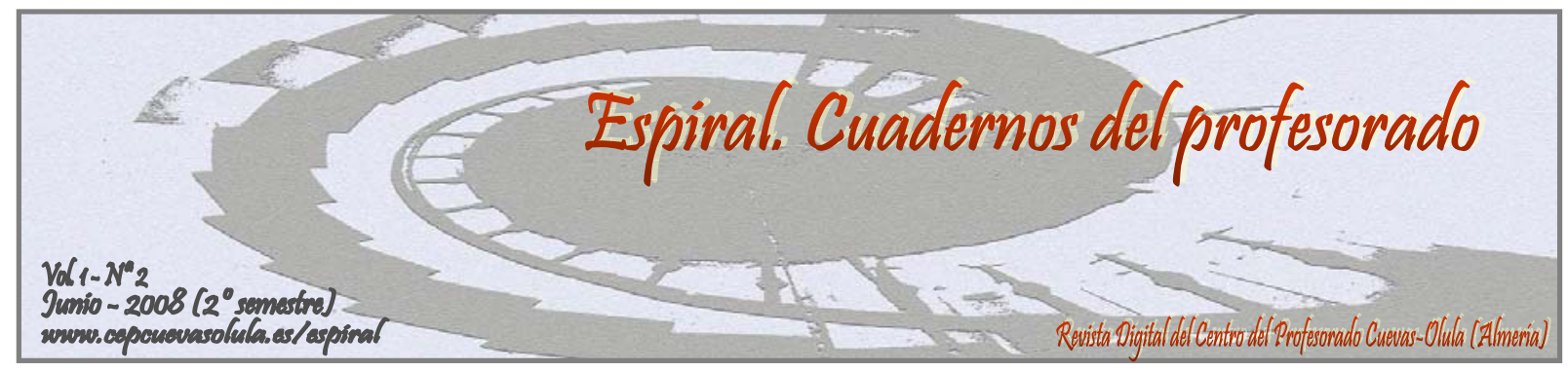

\title{
PERFIL DE LA UNIVERSIDAD PEDAGÓGICA EXPERIMENTAL LIBERTADOR COMO ORGANIZACIÓN QUE APRENDEN EN EL CONTEXTO UNIVERSITARIO VENEZOLANO
}

\author{
Yolibet Ollarves Levison y Luis Arturo Salguero
}

Universidad Pedagógica Experimental Libertador de Venezuela

Enviar correspondencia a: yolibetollarves@gmail.com

RESUMEN: Esta investigación tuvo como propósito presentar un análisis del perfil de la Universidad Pedagógica Experimental Libertador (UPEL), desde la perspectiva de las organizaciones que aprenden descritas por Peter Senge. La metodología utilizada se fundamentó en una investigación documental (UPEL, 2004) apoyada en el análisis de contenido mediante la técnica Krippendorff. Para el logro de este objetivo se realizó una revisión teórica de la concepción de: (a) las universidades como organizaciones que aprenden y, (b) el talento humano en las universidades como determinantes del éxito organizacional. Como aportes, este trabajo proporciona insumos teóricos que permiten redefinir el rol de la UPEL y del docente universitario frente a los desafíos del subsistema de educación superior, entre ellos optimizar la calidad y la productividad de la universidad en un contexto de aprendizaje y globalización cada vez más cambiante, así como dar respuesta a las distintas problemáticas existentes desde una óptica de pertinencia social.

Palabras clave: Universidad, Organizaciones que aprenden, Talento Humano, Docente Universitario.

ABSTRACT: This investigation had as purpose to present an analysis of the profile of the University Pedagogic Experimental Liberator (UPEL), from the perspective of the organizations that learn described by Peter Senge. The used methodology was based in a documental investigation (UPEL, 2004) supported in the content analysis by means of the technical Krippendorff. For the achievement of this objective was carried out a theoretical revision of: (a) the conception of the universities like organizations that you learn and (b) the human talent in the universities like decisive of the organizational success. Ace contributions, this work provides theoretical inputs that allow to redefines the list of the UPEL and of the educational university student in front of the challenges of the subsystem of superior education, among them to optimize the quality and the productivity of the university in to learning context and lives changing globalization, ase well ase to give answer to the different ones problematic existent from an optics of social relevancy it lives.

Key words: University, Organizations that learn, Talent Human, Educational University student.

\section{1.- INTRODUCCIÓN.}

El reconocimiento de la responsabilidad de los docentes como agentes de la transmisión cultural y social del conocimiento, capaces de comprender y operar reflexivamente sobre el mundo, obliga a las universidades a replantear la dinámica organizacional del proceso de desarrollo profesional de sus docentes como eje estratégico con miras a superar las debilidades e impulsar las fortalezas en pro de su mejora, dado 
que definitivamente ejerce un impacto en la transmisión y producción del conocimiento y la cultura de un país, y para ello es fundamental que la organización, en este caso, la universidad, sustentada en una paradigma de cambio organizacional, se conozca a sí misma con miras a impulsar estrategias que fortalezcan sus procesos medulares de docencia, investigación y extensión, así como la productividad y desempeño de sus miembros, lo cual definitivamente ejercerá una influencia positiva en el contexto local, regional y nacional.

Por lo antes expuesto, debe partirse de la premisa de que el cambio ha de convertirse en una constante y el debate profesional en el escenario de reflexión para evaluar el rol de la universidad venezolana con relación a las demandas derivadas de estos tiempos y de las circunstancias y condiciones que enmarcan su entorno inmediato. De allí que el propósito de este estudio fue analizar el perfil de la Universidad Pedagógica Experimental Libertador, desde la perspectiva de las organizaciones que aprenden descritas por Peter Senge, considerando que las universidades son fuentes de saber, generadoras de conocimiento que demandan en su estructura y funcionamiento una serie de características que los individuos y colectivos, pueden contribuir a construir al establecerse un camino orientado al autoconocimiento, al trabajo en equipo, la innovación, la investigación, la creatividad, el diálogo y el sentido de compromiso e identidad profesional y organizacional, como aspectos claves de una visión compartida que guía su ser y su quehacer.

\section{2.- LAS ORGANIZACIONES EDUCATIVAS EN UN CONTEXTO DE APRENDIZAJE}

"Todas las organizaciones están perfectamente
diseñadas para obtener los resultados que
obtienen”
(Henry Mintzberg)

Las organizaciones, asumidas como colectivos sociales formalmente establecidos, plantean a lo largo de la historia una revisión de su ser y de su quehacer, por lo tanto, no es de extrañar que se haya institucionalizado el tema como eje de vital interés en los actuales momentos. Para comprender el comportamiento de las organizaciones, debe estudiarse a partir de sus componentes, como lo señala Chiavenato
(2002), al referirse al comportamiento de personas y grupos, estructura de las organizaciones y procesos que dan vida a la organización.

De allí se destaca la importancia de coordinar el comportamiento y satisfacer las necesidades de dichas personas, mediante el trabajo en equipo, la colaboración, la comunicación abierta y el liderazgo, con base a las actividades de planificación, organización, dirección y control. En este sentido, Ramírez (2002), Robbins (1997) y Daft y Steers (1992) coinciden en definir a las organizaciones como entidades sociales que existen cuando un conjunto de individuos trabajan en común en el cumplimiento de ciertas metas que no podrían alcanzar solos, es decir, que cada organización difiere respecto de sus metas en función de la naturaleza de origen.

Por consiguiente, si las organizaciones surgen de la necesidad de lograr objetivos comunes, entonces, Chiavenato (2003) plantea tres razones que explican su existencia, a saber: (a) sociales: por su característica gregaria al requerir relacionarse unos con otros; (b) materiales, asociadas al incremento de habilidades, la reducción de tiempo requerido para alcanzar una meta y la acumulación de conocimientos para lograr una comunicación efectiva con los demás miembros; y por (c) efecto sinérgico: producto del efecto generado por el trabajo en conjunto, el cual es mayor que el efecto derivado de la suma de la producción individual.

Cabe destacar la definición expuesta por Senge (1995) en su libro "La Quinta disciplina”, el cual describe a las organizaciones en aprendizaje como lugares:

"... donde las personas amplían continuamente su capacidad de crear los resultados que verdaderamente desean, donde se nutren nuevas $y$ amplias formas de pensar, donde se deja libre la aspiración colectiva y donde la gente continuamente está aprendiendo cómo aprender en conjunto" (p. 268).

Este enfoque considera que todos los miembros de la organización son elementos capaces de aportar y valorar, adoptándola como propia y actuando con responsabilidad; es decir, que son capaces de tomar decisiones, de enriquecer esa visión haciendo uso de su 
creatividad, reconociendo sus propias cualidades y limitaciones y aprendiendo a crecer a partir de ellas. Por consiguiente, lo esencial en organizaciones insertadas en un contexto de aprendizaje es el proceso de aprendizaje en la organización, lo cual para Senge (1995) requiere de la interacción de cinco aspectos fundamentales:

1. Pensamiento Sistémico, gracias al cual se integran las otras disciplinas y se generan los marcos de referencia considerando la totalidad de los acontecimientos, preocupación por los procesos y no por tratar los problemas como establecimientos estancados.

2. Dominio Personal, que permite aclarar y ahondar continuamente la visión personal, ampliar la propia capacidad de juicio y actuación, y avanzar en visiones objetivas. Condiciones adecuadas relacionadas al desarrollo personal y profesional.

3. Comprensión de los Modelos Mentales, que facilita desmenuzar las nociones y supuestos arraigados, generalizaciones e imágenes que influyen sobre la forma de ver el mundo. La apertura a nuevos planteamientos es una exigencia.

4. Construcción de una Visión Compartida entre las personas, como base del análisis, proporciona coherencia en todas las actividades.

5. Aprendizaje en Equipo, que supone la capacidad de los miembros del equipo para suspender los supuestos arraigados e ingresar en el pensamiento conjunto.

En resumen, en una organización el dominio personal comienza por aclarar los intereses, pues está asociado al conocer, entonces incluye el sentido de pertenencia, el compromiso, el sentido de misión. Aquí se dan las conexiones entre aprendizaje personal y aprendizaje organizacional, los compromisos recíprocos entre individuo y organización. Sin embargo, existen supuestos arraigados en la cultura organizativa que influyen en el comportamiento de sus miembros y de la propia organización, y es lo que se denomina paradigmas o modelos mentales, los cuales se presentan en individuos y organizaciones, pero que son modificables mediante el aprendizaje institucional en el cual se busca sacar del interior de los componentes de la organización, las ideas, prácticas y pensamientos ocultos, que puedan afectarla favorable o desfavorablemente. Se trata entonces de sumar las visiones individuales a una sola visión institucional y compartir, por lo que es importante construir una visión consensuada, capaz de crear identidad. De allí la relevancia del aprendizaje en equipo, el cual se inicia con el diálogo o la capacidad de aprender a reconocer patrones de interacción que erosionan el aprendizaje del equipo, ejercitando la capacidad de los integrantes para suspender los supuestos e ingresar a un auténtico "pensamiento conjunto".

No obstante, todos estos aspectos mencionados deben fundamentarse en la integración y la retroalimentación, y es allí donde aparece el pensamiento sistémico para organizar y coordinar la acción de cada elemento como parte de un todo.

Por consiguiente, la existencia de todos estos elementos constituyen una guía o quizás un camino hacia el cual se puede transitar, pues la integración sistémica y sinérgica de sus componentes optimiza el desarrollo de una organización y de sus miembros; sin embargo, Senge (1992) sostenía que las organizaciones que aprenden se caracterizan por tener institucionalizados procesos de reflexión y aprendizaje intrínseca en la planificación y evaluación de sus acciones, lo cual les permitía adquirir una nueva competencia: aprender cómo aprender; lo que implica transformar los "modelos mentales" vigentes, así como generar "visiones compartidas". Argyris (1993, p. 85), por su parte, subrayó que el aprendizaje organizativo implica la capacidad de aprender de los errores, contribuyendo, "en lugar de una adaptación, nuevas soluciones, por alteración, en un ciclo doble y no simple, de los marcos mentales en los que hasta ahora ha funcionado su acción”.

Así pues, una organización que aprende es aquella que tiene una competencia nueva; que le capacita para que aprendiendo de la experiencia pasada y presente, sea capaz de procesar la información, corregir errores y resolver sus problemas de una manera creativa o transformadora, no meramente de modo reproductivo.

\section{3.- PERFIL DE LAS UNIVERSIDADES COMO ORGANIZACIONES QUE APRENDEN.}

De lo anteriormente planteado se desprende la existencia de un perfil de las universidades como organizaciones que aprenden, sin embargo es importante resaltar que en los artículos 1,2 y 3 de la Ley de Universidades (1970) venezolana se distinguen las directrices conceptuales que 
definen a las universidades como una: "comunidad de intereses espirituales que reúne a profesores y estudiantes en la tarea de buscar la verdad y afianzar los valores trascendentales del hombre”, pero que además son Instituciones al servicio de la Nación y a ellas corresponde colaborar en la orientación de la vida del país mediante su contribución doctrinaria en el esclarecimiento de los problemas nacionales y como función rectora se establece en su Artículo 3 que:

Las Universidades deben realizar una función rectora en la educación, la cultura y la ciencia. Para cumplir esta misión, sus actividades se dirigirán a crear, asimilar y difundir el saber mediante la investigación y la enseñanza; a completar la formación integral iniciada en los ciclos educacionales anteriores; $y$ a formar los equipos profesionales y técnicos que necesita la Nación para su desarrollo y progreso (p.3).

De tal forma que para lograr cumplir con su misión, los actores sociales que forman parten de esta comunidad de intereses deben desarrollar un trabajo sinérgico para contribuir al logro de estos fines fundamentalmente universales y sociales. De allí la pertinencia de la teoría de Senge al ofrecer herramientas para consolidar en estos recintos universitarios un sistema que se ajuste a las necesidades sociales del país, contribuya con el progreso de la educación y certifique el avance de la ciencia y de la tecnología.

En el subsistema de educación superior venezolano, la Universidad Pedagógica Experimental Libertador (UPEL, 2007) de acuerdo a su misión es una universidad pública de alcance nacional e internacional, líder en la formación, capacitación, perfeccionamiento y actualización de docentes de elevada calidad personal y profesional, $\mathrm{y}$ en la producción y difusión de conocimientos socialmente válidos, que desarrolla procesos educativos con un personal competente, participativo y comprometido con los valores que promueve la Universidad. Asimismo, su visión se proyecta como una universidad de excelencia, comprometida con el país para elevar el nivel de la educación, mediante la formación de docentes competentes; capaz de generar conocimientos útiles para implementar procesos pedagógicos innovadores e impulsar la transformación de la realidad social, y así contribuir al logro de una sociedad más próspera, equitativa y solidaria. Cabe destacar que está conformada por ocho institutos pedagógicos, ubicados en casi todos los estados del país.

De acuerdo con los postulados de Senge (1995), los aspectos que describen una organización, en este caso la universidad pedagógica desde la concepción del aprendizaje, serían los siguientes:

1. Compartir una visión: al comprender su contexto, las universidades podrán construir y proyectar el futuro a partir de: (a) análisis y discusión sobre el complejo contexto educativo, el cual posee diversos niveles y modalidades; (b) generación de una cultura centrada en principios $y$ valores de flexibilidad, creatividad y productividad intelectual; y (c) evaluación de sus productos y servicios con relación a otras universidades e institutos educativos, para lo cual puede apoyarse en sus procesos de evaluación institucional; y entre las dificultades que deben superar se destacan: (a) un clima sustentado en la desmotivación o desconfianza de los colaboradores, lo cual no fortalece el diálogo e intercambio necesario entre sus miembros; (b) la tendencia del nivel estratégico a imponer ideas, derivada de una práctica gerencial tradicional jerárquica y matricial; y (c) la necesidad de obtener respuestas rápidas.

2. Actuar mediante un sistema de corresponsabilidad y compromiso mutuo: Cuando los docentes como líderes influencian estimulando la creatividad, la autonomía y la libertad en sus cátedras y departamentos, así como en sus unidades de investigación ayudan a compartir la visión creando valores compartidos y estimulando el aprendizaje, facilitando un sistema universitario que permita captar la innovación a través de la comunicación, el pensamiento colectivo e institucional. Por consiguiente, para alcanzar este sistema es importante: (a) estimular a los actores sociales, en este caso a la comunidad universitaria hacia la autonomía y la autogestión; (b) reconocer la experticia, el saber y la necesidad de aprender en todos los niveles de la organización, lo cual demanda de un proceso de transformación interna que permita derribar las barreras comunicacionales existentes; (c) incluir a todos a compartir los éxitos, beneficios y fracasos de su acción educativa, de vital relevancia porque favorece la cohesión interna y la identidad profesional y organizacional. Sin embargo, entre las dificultades que deben superar están las 
siguientes: (a) visiones de liderazgo tradicional; (b) adaptación a rol de hacedores antes que pensadores; y (c) temor a asumir los riesgos de las propias decisiones.

3. Creer en su Potencial como Equipos: Los docentes aprenderían mejor en grupos, en redes educativas, investigativas o de innovación, en las cuales se compartan colectivamente la información, los principios y valores comunes a todos los miembros, vale decir, donde se gestione el talento humano de sus miembros, tal es el caso de los departamentos, los núcleos, centros o institutos de investigación, cuyo potencial se proyecta en la medida en que se fortalece el colectivo y demanda que conjuntamente: (a) piensen y analicen las situaciones en equipo; (b) se integren en forma multidisciplinaria; y (c) promuevan la solución de problemas entre todos. No obstante las dificultades a superar son: (a) tendencia al individualismo, lo cual es el modelo de formación que predomina en la universidad; (b) un proceso de toma de decisiones con el uso de informaciones parciales, donde se puede atentar con la armonía necesaria en un clima organizacional, debido al alto nivel de incertidumbre y falta de asertividad; y (c) un nivel mínimo de participación de parte de los colaboradores, quienes son piezas importantes del proceso.

4. Compartir la Información y las Ideas: Cuando los miembros de una universidad comparten las experiencias, ideas, pensamientos y proyectos esenciales que dan sentido a su razón de ser y a su visión, la capacidad de rendimiento y de respuesta es muy alta y optimiza el tiempo. Cuenta con (a) su sistema de comunicación es bastante flexible y abierto, considerando que esta universidad agrupa a ocho institutos pedagógicos distribuidos a nivel nacional; (b) trabajo coordinado y difundido; y (c) generación de creencias compartidas que facilitan el respeto y entendimiento mutuo, lo cual se materializa a través de sus valores organizacionales: calidad, defensa del ambiente, equidad, sentido de pertenencia, respeto, responsabilidad, sentido crítico, solidaridad e innovación; los cuales emergieron durante la formulación Plan de Desarrollo 2007-2011. No obstante, deben superar: (a) la falta de un sistema de comunicación fluido considerando las diferencias geográficas; (b) pasividad informativa, al no contar con una plataforma tecnológica y comunicacional más efectiva y eficiente; y (c) la imposición de ideas de algunos grupos de poder que representan los vestigios de la tradición tradicional y falta de compromiso de algunos miembros de la universidad.

5. Avances apoyados en el aprendizaje. Asumen la idea de que el aprendizaje fluye libremente en todos los niveles de la organización hacia quien lo necesite, hay facilidades para crear, adquirir y transferir el conocimiento, lo cual se verifica por la existencia de una estructura organizativa que intenta impulsar y garantizar las funciones universitarios, el rol social de sus docentes y la pertinencia del perfil de sus egresados. En tal sentido: (a) generan y estimulan un clima de aprendizaje en general; (b) coadyuvan al desarrollo personal y profesional de sus miembros en forma individual y colectiva; y (c) transfieren el conocimiento a otros y modifican su comportamiento generando nuevos conocimientos. No obstante, tienen dificultades que superar, tales como: (a) falta de habilidades para asumir un proceso de aprendizaje constante; (b) tendencia de los niveles estratégicos y tácticos a controlar; y (c) falta de compromiso como corresponsable de la labor social y educativa de la universidad.

6. Transformarse: Innovar, Inventar $y$ Cambiar: la comunidad de intereses se orienta hacia la búsqueda de nuevas ideas y experiencias, conscientes de la necesidad de un cambio a tono con los nuevos tiempos y la mejora continua, lo que conlleva a probar nuevas formas de organizarse internamente como universidad. De tal manera que: (a) cuestionan lo presente, exploran nuevas ideas y oportunidades en todos los niveles de organización; (b) intentan reducir las barreras y bloqueos para aprender individualmente y en el contexto, de allí que dentro de los ejes estratégicos que definen el actual plan de desarrollo, se cuentan la formación, el talento humano, la generación, divulgación y aplicación de conocimiento, la integración con la comunidad y la gestión; y (c) valorar y aceptar los aportes de cada quien, por lo cual se le dio participación desde su formulación. Sin embargo, debe superar: (a) la resistencia natural el cambio y la inercia de lo que se sabe y se hace bien; (b) la falta de voluntad y sinergia para resolver los conflictos y problemas, y (c) la tendencia a desestimar algunas ideas o propuestas alejadas de la visión construida.

Puede afirmarse que según la teoría de Senge, las universidades como organizaciones en un contexto de aprendizaje, están orientadas a la creación y transmisión de conocimientos y 
modelos mentales, que demandan de sus miembros compromiso y el desarrollo máximo de su potencial individual y profesional, lo cual es fundamental para alcanzar la eficacia profesional de sus miembros y la productividad organizacional universitaria. Por consiguiente, este enfoque permite entender el significado de organización que deben asumir las universidades en la actualidad, y avizora el rol activo que deben asumir sus distintos actores en forma individual y colectiva, y ser verdaderos impulsores de transformación social.

\section{4.- EL TALENTO HUMANO EN LAS UNIVERSIDADES.}

El mayor potencial que tiene cada persona para crecer está en aquello que le resulta natural, está en sus talentos.

(Pérez Segnin)

Quezada (2004, p. 1) asegura que en la actualidad puede redefinirse el Talento como "la capacidad puesta en práctica de un profesional o grupo de profesionales comprometidos que alcanzan resultados superiores en un entorno y en una organización determinados”. De lo anteriormente planteado surge la interrogante acerca del papel que juega la gestión de los recursos humanos en las organizaciones para lograr desarrollar el potencial de los talentos en forma individual, grupal y organizacional y así obtener determinados resultados.

$\mathrm{Al}$ respecto, cabe destacar que la gestión de los recursos humanos en una organización, según Chiavenato (2003), nace de las necesidades de disminuir el conflicto organizacional entre los objetivos organizacionales y los objetivos individuales de sus miembros. En este concepto se destacan tres aspectos fundamentales:

1. Las personas como seres humanos diferentes entre sí, dotados de personalidad propia, con una historia particular y diferenciada, poseedores de habilidad y conocimiento, destrezas y capacidades, indispensables para administrar de manera adecuada los recursos organizacionales.

2. Las personas como elementos impulsores de la organización, capaces de dotarla de la inteligencia, el talento y el aprendizaje indispensables para estimular la renovación y competitividad constantes en un mundo lleno de cambios y desafíos.

3. Las personas como socios de la organi- zación, capaces de llevarla a la excelencia y al éxito, que invierten esfuerzo, dedicación, responsabilidad y compromiso para obtener ciertas ganancias, ya sean salarios, incentivos, crecimiento profesional o de carrera.

En definitiva, la adopción de este esquema aporta elementos fundamentales para que cada miembro de la organización explore, desarrolle y valore su potencial individual, enmarcados en un contexto de aprendizaje en el que se asume el compromiso de dar valor agregado a la organización como parte de un binomio simbiótico, en el que ambos crecen, se favorecen y se nutren. Desde esta perspectiva, Argirys y Shön (1978), citado por Meléndez Ferrer (2004), plantean que la persona en la dinámica y desarrollo de la organización en la que labora tiende a demostrar una actitud positiva para planificar su teoría acción profesional, porque puede: (a) manifestar una actitud organizacional hacia la concienciación de ideas y sentimientos que generan su conducta académica, profesional y organizacional; (b) identificar las consecuencias organizacionales que ideas y sentimientos en la formación profesional alternativa; y (c) difundir los enfoques epistémicos que fundamentan las tendencias organizacionales presentes, que le dan contenido a su pensamiento-acción.

En el contexto universitario, según la Ley de Universidades (1970) en su artículo 83 se establece que "la enseñanza y la investigación, así como la orientación moral y cívica que la Universidad debe impartir a sus estudiantes, están encomendadas a los miembros del personal docente y de investigación”. Asimismo, prescribe la norma en su artículo 85 que para ser miembro del personal docente y de investigación se requiere: (a) poseer condiciones morales y cívicas que lo hagan apto para tal función; (b) haberse distinguido en sus estudios universitarios o en su especialidad o ser autor de trabajos valiosos en la materia que aspire a enseñar; y (c) llenar los demás requisitos establecidos en la presente Ley y los reglamentos.

En su artículo 86 también se prevé que "los miembros del personal docente $y$ de investigación se clasificarán en las siguientes categorías: miembros Ordinarios, Especiales, Honorarios y Jubilados".

En este sentido, los miembros del personal académico de la universidad constituyen su principal talento humano. Sin embargo, en el 
ejercicio de su rol profesional, se ve afectado por diversos factores individuales y organizacionales, lo cual puede incidir en su desempeño y en su rendimiento profesional. Para atender a estas circunstancias, cada vez es más ingente, que las universidades implanten estrategias de desarrollo y formación, continuas y dinámicas, que además de permitirle el ascenso en el escalafón universitario (Instructor, Asistente, Agregado, Asociado y Titular), le potencien en el proceso de agregar valor a través de sus productos intelectuales, para que logren impulsar las transformaciones necesarias a tono con los tiempos y las innovaciones. En este orden de ideas, para que los docentes logren alcanzar el perfil deseado es prioritario implementar dispositivos de formación que los comprometa a aumentar sus capacidades individuales y grupales en consonancia con la misión, visión y valores organizacionales, lo cual supone el acompañamiento de políticas, incentivos profesionales y planes integrales de formación y desarrollo que fortalezcan a su vez la capacidades organizativas de la propia universidad.

\section{5.- DISCUSIÓN.}

Cada ser humano es una obra maestra. (Henry Thoreau)

Para efectos de esta investigación se asume el concepto de universidad y particularmente, de la UPEL como una organización inteligente de tipo educativa en la que aprenden e interactúan sobre los actores sociales que la conforman y se orientan a través de sus funciones universitarias al logro de su misión y visión; y cuya ventaja competitiva es la capacidad de aprendizaje que garantiza su sustentabilidad en el tiempo, a través de la capacidad de adaptación y cambio, y de sus propósitos establecidos en la normativa vigente, específicamente:

1.- Formar, profesionalizar, perfeccionar, capacitar y actualizar ciudadanos para desempeñarse en el ámbito educativo, con una cultura investigativa, partícipes de una educación permanente que le permita la búsqueda, evaluación y aplicación de información pertinente, para posibilitar las transformaciones a partir de sus conocimientos; lo cual requiere de pensamiento sistémico, visión compartida y dominio personal.

2.- Educar y promover las comunidades del saber, los valores culturales, científicos y humanísticos que contribuyen al refuerzo de la propia identidad, de la esencia del ser, para partir de allí con el encuentro con lo global; significa entonces que a través del aprendizaje en equipo y un pensamiento sistémico pueden lograrlo.

3.- Constituir la pedagogía en un lugar privilegiado para el reencuentro con la ética, la estética y sus fundamentos y General los espacios que le permiten al hombre su aprecio por la cultura, el deporte y la recreación como formas esenciales de su realización plena; integrando la Docencia, la Investigación y la Extensión como un solo quehacer universitario, enriqueciéndose mutuamente. Al respecto sólo cabe destacar que mediante las herramientas de un pensamiento sistémico integrado a la visión compartida y a la acción de los modelos mentales efectivos, la universidad tiene una propuesta sugerente para adaptarse a los nuevos tiempos.

Con relación al talento humano clave para coadyuvar a la construcción de organizaciones inteligentes, el docente universitario se concibe como un ser personal y profesional que a través del desempeño de sus funciones universitarias (docencia, investigación, extensión y gestión), desarrolla su potencial y capacidad de aprendizaje para crear en forma individual o cooperativa una serie de productos intelectuales que contribuyen a elevar la calidad de su perfil profesional en función de la misión y visión de la universidad a la cual pertenece. Sin embargo, cuando se intenta definir el perfil que deben tener los docentes universitarios se encuentran diversas tendencias, por lo tanto es necesario considerar una posición que permita sin obviar la complejidad de dicho perfil profesional, plantear la relevancia de aspectos asociados a su autoestima, competencias profesionales, valores éticos, compromiso institucional y por supuesto todo lo que tiene que ver con las compensaciones $\mathrm{y}$ beneficios.

Igualmente, es importante destacar al resto de los miembros que conforman la comunidad universitaria, vale decir, personal administrativo, personal de servicio, estudiantes e incluso la comunidad, cuya participación activa y valor agregado contribuyen día a día con sus acciones a lograr las metas propuestas, y garantizan la pertinencia social de los planes y proyectos que se aspiran implementar en las áreas de docencia, investigación, extensión y gestión, por lo cual el seguimiento y la evaluación institucional se convierten en herramientas de mejora continua. 


\section{6.- CONCLUSIONES.}

Actualmente la UPEL, como parte de un contexto de aprendizaje y creatividad organizacional, debe desarrollar su capacidad de aprendizaje y de adaptación interna para lograr ajustarse a las exigencias de un mundo organizacional altamente cambiante y desarrollar una visión tal en la gestión de sus recursos humanos, específicamente de sus docentes universitarios, que le permita a la institución definir y fortalecer sus propósitos de desarrollo profesional a partir del ingreso de su personal académico, ya que se evidencia la ausencia de fines orientados a direccionar dicho proceso. En este orden de ideas, es menester plantearse a partir de las políticas y estrategias prescritas, la promoción de un proceso de formación planificado desde la inducción e integración, así como de procesos de socialización mediante la cultura y subculturas de la comunidad universitaria, que incluya además procesos de preparación u orientación, capacitación y actualización para su talento humano en ocasión de nuevos retos y desempeños funcionales que habrán de asumir y fundamentalmente, que logre impulsar efectos sinérgicos entre sus miembros sobre la base de las potencialidades individuales, grupales e institucionales.

Finalmente, se plantea la necesidad de coordinar esfuerzos con otros colegas interesados en el tema para luego crear una línea de investigación denominada: Cambio, Universidad y Pertinencia social, considerando que es uno de los pilares fundamentales para generar los cambios y transformaciones que requiere el subsistema de Educación Superior y más específicamente en la UPEL en la actualidad, porque deben generarse espacios para la disertación, reflexión, divulgación y difusión de temas de interés para la universidad.

\section{7.- REFERENCIAS BIBLIOGRÁFICAS.}

Argyris, C. (1993). Cómo vencer las barreras organizativas. Madrid: Díaz de Santos.

Chiavenato, I. (2002). Administración de los Recursos Humanos. Bogotá: McGraw Hill.

Chiavenato, I. (2003). Gestión del Talento Humano. Bogotá: Mc Graw Hill.

Constitución Bolivariana de Venezuela (1999). Gaceta Oficial de la República Bolivariana de Venezuela, 5453, marzo 1999.
Daft, R. \& Steers, M. (1992). Organizaciones. El Comportamiento del individuo y de los grupos humanos. México: Limusa.

Ley de Universidades (1970). Gaceta Oficial de la República de Venezuela, 1429, 8 de septiembre (Extraordinario).

Melendez Ferrer, L. (2004). Actitud organizacional del Profesor Universitario. Revista Venezolana de Gerencia, 9 (26) 354-374.

Quezada M., H. (2004). El Talento en las organizaciones. Revista Electrónica Gestiopolis [ON LINE], 8. Disponible en http: www.gestiopolis.com. [Consulta: 2005, mayo 3].

Ramírez, C. (2002). La Gestión Administrativa en las Instituciones Educativas. México: Editorial Trillas.

Robbins, S. (1997). Comportamiento Organizacional. Conceptos Controversia y Aplicaciones. México: Editorial Prentice Hispanoamericana.

Senge, P. (1992): La Quinta Disciplina. El arte y la práctica de la organización abierta al aprendizaje. Barcelona: Granica.

Senge P. (1995). La Quinta disciplina: las organizaciones que aprenden. Barcelona: Granica.

Universidad Pedagógica Experimental Libertador (2004). Plan de Desarrollo 2007-2011. Caracas: Dirección de Planificación y Desarrollo de la UPEL.

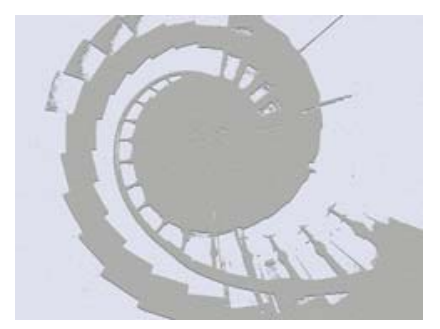

